\title{
Protection against Toxoplasmosis in Mice Immunized with Different Antigens of Toxoplasma gondii Incorporated into Liposomes
}

\author{
Mohamed MA Elsaid, Ricardo WA Vitor, Frederic JG Frézard*, \\ Maria S Martins/ ${ }^{+}$
}

Departamento de Parasitologia *Departamento de Fisiologia, Instituto de Ciências Biológicas, Universidade Federal de Minas Gerais, Caixa Postal 486, 31270-901 Belo Horizonte, MG, Brasil

Different toxoplasma antigens were entrapped within liposomes and evaluated, in this form, for their ability to protect Swiss mice against toxoplasma infection: soluble tachyzoite antigen (L/TAg), tissue cyst (L/CAg), tachyzoite plus tissue cyst (L/TCAg) or purified antigen of tachyzoite (L/pTAg). The protein used in L/pTAg was purified from tachyzoites using a stage-specific monoclonal antibody which reacted at a molecular weight of $32 \mathrm{kD}$ in SDS PAGE and silver stain using reduced condition. To compare the immuno-adjuvant action of liposomes and of Freund's Complete Adjuvant (FCA), another group of mice was immunized with soluble tachyzoite antigen (STAg) emulsified in FCA (FCA/TAg). Control groups were inoculated with (STAg) alone, phosphate-buffered saline (PBS), FCA with PBS (FCA/PBS) and empty liposomes (L/PBS). Mice were inoculated subcutaneously with these antigens six, four and two weeks before a challenge with 80 tissue cysts of the P strain of Toxoplasma gondii orally. All mice immunized with or without adjuvant showed a humoral response, as measured by Elisa. However, no correlation was found between antibody titer and protection against the challenge. All mice immunized with L/pTAg or L/TCAg survived (100), whereas $80 \%$ and $90 \%$ of mice from groups which received respectively $P B S$ or FCA/PBS and L/PBS died. All mice immunized with antigens entrapped within liposomes (L/TAg, L/CAg, L/TCAg and L/pTAg) showed low numbers of intracerebral cysts.

Key words: toxoplasma - immunoprotection - purified antigen - mice - Brazil

Toxoplasma gondii is an obligate intra-cellular parasite, affecting a wide range of animals, including humans. Infection consists of a transient acute phase caused by proliferative tachyzoites followed by the formation of dormant tissue cysts containing bradyzoites. The disease can be serious if acquired congenitally (Wong \& Remington 1994) or in immunosuppressed individuals, particularly patients with acquired immunodeficiecy syndrome (Aids) (Luft et al. 1993).

Toxoplasmosis, in an immunocompetent host, leads to the induction of a life=long protective immunity against illness due to reinfection. These privileged protective antigens are candidates for the development of a vaccine strategy (Alexander et al. 1993).

\footnotetext{
Work supported by Fapemig, MG, Brazil.

${ }^{+}$Corresponding author. Fax: +55-31-499.2970. E-mail: msonia@icb.ufmg.bar

Received 13 October 1998

Accepted 19 April 1999
}

Vaccination attempts with live, attenuated, killed or lysed parasites, as well as different antigenic fractions of the parasite, have been conducted with varying success (Araújo 1994). Vaccines with live organisms are currently in use (Buxton 1993, Dubey et al. 1994) but they represent a potential hazard. However, the development of vaccines in the future will not only have to take into account all life-cycle stages that need to be targeted but will also have to consider which immune responses need to be generated and in which tissue sites.

The induction of protective immunity is dependent on the nature of the immune response to be generated. Antibodies have been shown to transfer protection passively (Johnson et al. 1983). There is also evidence that protective immunity against toxoplasmosis can be mediated by CD8 and CD4 lymphocytes (Gazzinelli et al. 1998). Thus, an adjuvant that promotes activation of these Tcell subsets would theoretically be an ideal component of a $T$. gondii vaccine.

Freund's complete adjuvant has been used in a number of studies (Kasper et al. 1985, Alexander et al. 1993). On the other hand, administration of purified $T$. gondii antigen in the encapsulated form in liposomes (Bulow \& Boothroyed 1991) or as 
non-ionic surfactant vesicles (Alexander et al. 1993), was found to enhance-protection against infection by $T$. gondii in mice. Immunization with Escherichia coli expressed recombinant SAG1 (Petersen et al. 1998) or with STAg, together with cytokine interleukin-12 (Yap et al. 1998), induce partial protection against lethal infection with $T$. gondii in mice.

In this study, we determined the ability of different soluble antigens of $T$. gondii incorporated into liposome vesicles to induce protective immunity responses in mice against a natural route of infection, namely oral infection with tissue cysts.

\section{MATERIALS AND METHODS}

Mice - Six-eight week-old female Swiss mice were obtained from the experimental animal breeding laboratory of the Federal University of Minas Gerais (UFMG), divided in nine groups of ten animals.

Parasites - Cysts were obtained from the brain tissue of Swiss mice infected two months previously with $T$. gondii $\mathrm{P}$ strain of low virulence or C4 virulent strain isolated from dogs in São Paulo, Brazil (Jamra \& Vieira 1991). For antigen preparation, tissue cysts from the brain tissue of mice infected with the $T$. gondii $\mathrm{C} 4$ strain were purified on Dextran 30\% (Kasper 1989). Purified cysts were sonicated using ten periods of $30 \mathrm{~s}$ at $40 \mathrm{hertz}$ at 1 min intervals. The supernatant was collected following centrifugation at $4^{\circ} \mathrm{C}(30 \mathrm{~min} / 13,000 \mathrm{~g})$ and used as soluble antigen (S/CAg) in immunoprotection studies. Cysts of the P strain were used for oral-challenge infection of immunized mice.

The $\mathrm{N}$ strain was isolated from a rabbit in São Paulo (Nóbrega et al. 1952), and was maintained by serial passage in the peritoneal cavity of mice. Tachyzoites obtained by intraperitoneal washing were sonicated using five periods of $30 \mathrm{~s}$ at 40 hertz at $1 \mathrm{~min}$ intervals. The supernatant was collected following centrifugation at $4^{\circ} \mathrm{C}(30 \mathrm{~min} / 13,000 \mathrm{~g})$ and used as soluble antigen for purification of tachyzoite antigen (pTAg) and soluble tachyzoites antigen (STAg).

Production of monoclonal antibody (mAb) Tachyzoites were obtained from mice infected with T. gondii $\mathrm{N}$ strain and extracted with SDS sample buffer and electrophoresed on $12.5 \%$ gels. The portion containing proteins from $25-35 \mathrm{kDa}$ was cut and the tachyzoite antigen, eluted with ammonium bicarbonate/SDS buffer, was used to immunize $\mathrm{Balb} / \mathrm{c}$ mice. Splenocytes were fused with SP2/0 myeloma cells. The antibodies producing hybrids were screened by Elisa using tachyzoites or bradyzoites antigens to coat the plates. MAb producing cells were cloned twice limiting dilution. Specificity for the tachyzoite antigen was deter- mined by demonstration of reactivity on Western blots of T. gondii lysates (Fig. 1), a positive reaction with tachyzoite antigen in Elisa, but a negative reaction with the bradyzoite antigen in Elisa.

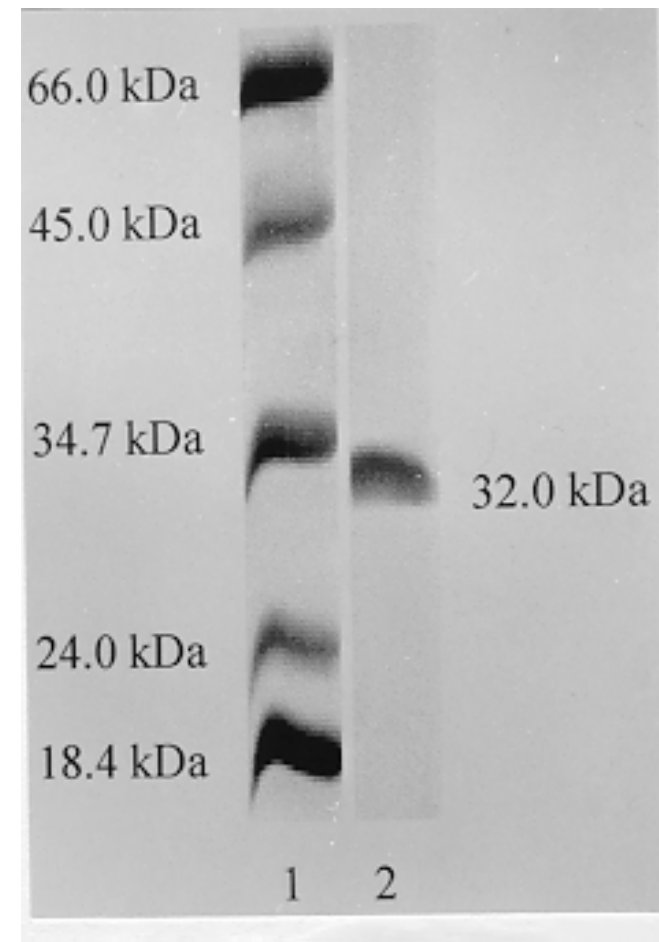

Fig. 1: electrophoretic profile (SDS-PAGE 12.5\%) of purified antigen of Toxoplasma gondii tachyzoites stained with silver (lane 2). Molecular weight markers are shown in lane 1.

Purification of tachyzoite antigen - Tachyzoite antigen was purified by immunoaffinity, as previously described (Bulow \& Overath 1986). Briefly, $\mathrm{N}$ strain STAg was applied to an affinity column of the anti-P32 mAb (4C3H4) coupled to cyanogen bromide-activated Sepharose 4B, as recommended by the manufacturer (Pharmacia). After application of the antigen, the column was sequentially washed with PBS. Bound P32 was eluted with $0.1 \mathrm{M}$ glycine ( $\mathrm{pH} 2.5$ ). Immediately after elution, fractions were neutralised with Trisma base (tris) salt. Fractions were analyzed by SDS-PAGE and silver staining for the presence of P32 (Fig. 2 ). We purified one protein with a molecular weight of $32 \mathrm{kDa}$. It is exposed in the tachyzoite but not in the bradyzoite by Elisa.

Multilamelar vesicles (MLVs) - A chloroform solution of an equimolar mixture of $\mathrm{L}-\alpha$ distearoylphosphatidycholine (DSPC, Sigma Chemical Co.) and cholesterol (CHOL, Sigma) was placed in a round-bottomed flask. After removal of most of the chloroform under a stream of nitro- 


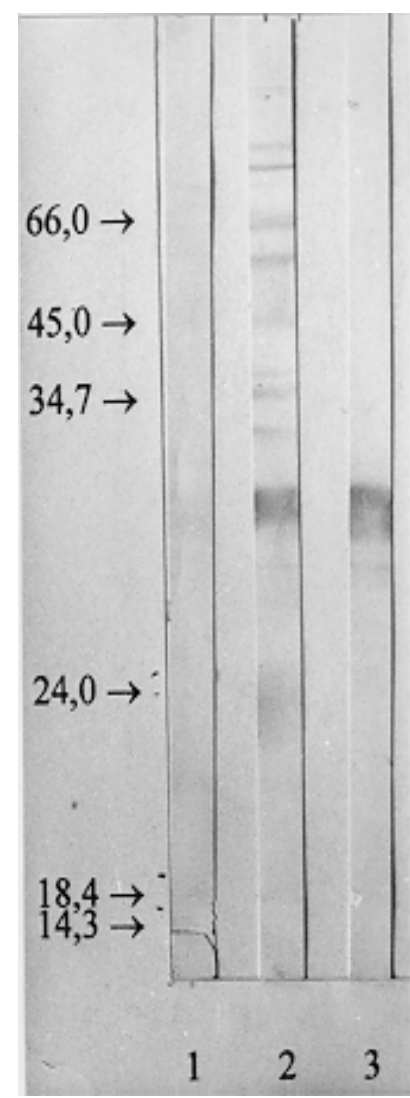

Fig. 2: immunoblotting characterization of Toxoplasma gondii tachyzoites lysate transferred on to nitrocellulose. Lane 1: normal mouse serum; lane 2: serum from mouse chronically infected with $T$. gondii; lane 3 : anti-tachyzoites monoclonal antibody $(4 \mathrm{C} 3 \mathrm{H} 4)$.

gen, the residual solvent was eliminated for $2 \mathrm{hr}$ with a rotary evaporator. MLVs were produced by hydration of the thin lipid film with distilled water for $1 \mathrm{hr}$ at $55^{\circ} \mathrm{C}$. The final lipid concentration was $45 \mathrm{mM}$.

Small unilamellar vesicles (SUVs)- SUVs were prepared by sonication of MLVs at $55^{\circ} \mathrm{C}$ using a $3 \mathrm{~mm}$ probe-sonicator (Misonix, ultrasonic liquid processor). Remaining MLVs and contaminating metal from the probe were eliminated by centrifugation at $10,000 \mathrm{~g}$ for $10 \mathrm{~min}$.

Dehydration rehydration vesicles (DRVs) - The general procedure for the entrapment of protein with DRVs has been described by Kirby and Gregoriadis (1984). Briefly, $1 \mathrm{ml}$ of SUVs was mixed with the following quantities of $T$. gondii: $400 \mu \mathrm{g}$ of STAg, $400 \mu \mathrm{g}$ (60,000 cysts) of CAg, $200 \mu \mathrm{g}$ STAg $+200 \mu \mathrm{g}$ CAg (30,000 cysts), or 50 $\mu \mathrm{g}$ of P32. The mixture was immediately frozen and then dried overnight. Rehydration of the dried powder was performed at $55^{\circ} \mathrm{C}$ as follows: onetenth of the original SUVs volume of distilled water was added, and the mixture was vortexed and incubated $\left(30 \mathrm{~min}, 55^{\circ} \mathrm{C}\right) ; 0.1$ volume of PBS was similarly added, and the mixture was vortexed prior to the addition of 0.8 volume of PBS and incubation $\left(30 \mathrm{~min}, 55^{\circ} \mathrm{C}\right)$. Antigens-containing DRVs were separated from free antigens by washing DRVs three times in PBS with centrifugation (43,000 g, $\left.30 \mathrm{~min}, 4^{\circ} \mathrm{C}\right)$. The liposome pellet was finally resuspended in PBS at a DSPC concentration of $18 \mathrm{~g} / \mathrm{l}$. Parallel preparations of empty (PBSloaded) DRVs were also made. The fraction of protein entrapped in liposomes was determined indirectly by protein titration in the supernatant of the first centrifugation (Bradford 1976).

Immunization and challenge of mice - Animals were immunized subcutaneously with three inoculations at 2-week intervals using different type of antigens of T. gondii (Table I). Each of the three control groups were inoculated with PBS, FCA with PBS, and liposome (L) with PBS. All mice were challenged with 80 cysts of P stain (LD90) orally 10 days after the final immunization. At the end of the experiment, the animals surviving at day 45 after infection were evaluated. All surviving animals were killed and the number of cysts was calculated.

Immune response in immunized mice - All mice were bled before immunization and seven days after the third immunization. Antibodies were determined by enzyme-linked immunosorbent assay (Elisa), using tachyzoite antigen soluble to coat a microplate.

TABLE I

Immunization of mice with different antigens of Toxoplasma gondii

\begin{tabular}{lcccccc}
\hline Dose/mouse & L/TAg & L/CAg & L/TCAg & L/pTAg & STAg & FCA/TAg \\
\hline 1 & $40 \mu \mathrm{g}$ & 2000 cysts & 1000 cyst $+20 \mu \mathrm{g} \mathrm{STAg}$ & $5 \mu \mathrm{g}$ & $40 \mu \mathrm{g}$ & $40 \mu \mathrm{g}$ \\
2 & $40 \mu \mathrm{g}$ & 2000 cysts & 1000 cyst $+20 \mu \mathrm{g}$ STAg & $5 \mu \mathrm{g}$ & $40 \mu \mathrm{g}$ & $40 \mu \mathrm{g}$ \\
3 & $40 \mu \mathrm{g}$ & 2000 cysts & 1000 cyst $+20 \mu \mathrm{g}$ STAg & $5 \mu \mathrm{g}$ & $40 \mu \mathrm{g}$ & $40 \mu \mathrm{g}$ \\
\hline
\end{tabular}

L/TAg: soluble tachyzoites antigen in liposomes; L/CAg: soluble tissue cysts in liposomes; L/TCAg: tachyzoites plus tissue cysts in liposomes; L/pTAg: purified antigen in liposomes; STAg: soluble tachyzoites alone; FCA/TAg: soluble tachyzoites with Freund's complete adjuvant. 
Statistical analysis - The survival rate was evaluated by the Qui-squared test. The mean number of cysts in the brain tissue for each group and the means of absorbance of Elisa were examined using the Duncan test (Duncan 1955) at 5\% level of probability.

\section{RESULTS}

Antibody response - The immunization of mice with toxoplasma antigen, with or without adjuvant, resulted in significant antibody production (Table II) when compared with non immunized groups. The antibody level was significantly higher in mice immunized with combined tachyzoite/cyst antigen incorporated into liposomes than in other groups.

Protection of mice after immunization - The protection afforded by the different types of immunization are presented in Fig. 3. Two (20\%) of the control mice injected with PBS with or without liposomes and one mouse (10\%) injected with FCA alone survived. All mice (100\%) immunized with L/pTAg or L/TCAg survived. Only 9 (90\%), $7(70 \%), 5(50 \%)$ and $4(40 \%)$ of the mice immunized with L/TAg, L/CAg, STAg and FCA/TAg respectively, survived. There are statistically significant differences in the degree of protection between mice immunized with L/TCAg and L/pTAg when compared with the control mice $(\mathrm{P}<0.05)$. There is also a significant difference between these two groups (L/TCAg and L/pTAg ) and mice immunized with STAg or FCA/TAg $(\mathrm{P}<0.05)$, and a significant difference was found between mice immunized with $\mathrm{L} / \mathrm{TAg}$ when compared with mice immunized with FCA/TAg $(\mathrm{P}<0.05)$.

The number of brain cysts was evaluated as an indicator of protection at the chronic stage of infection. As shown in Table II, low numbers of brain

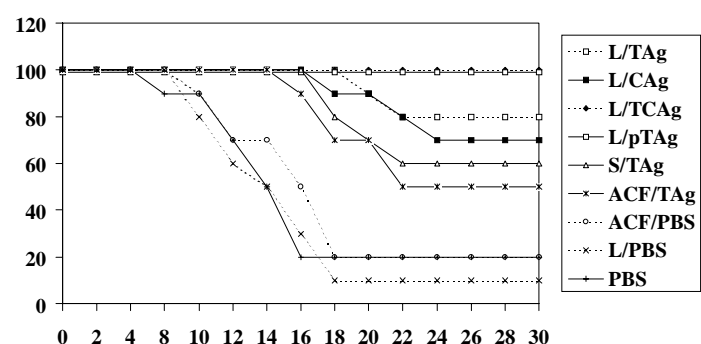

Fig. 3: survival of immunized mice after oral infection with 80 P strain cysts of Toxoplasma gondii. L/TAg: soluble tachyzoites antigen in liposomes; L/CAg: soluble tissue cysts in liposomes; L/TCAg: tachyzoites plus tissue cysts in liposomes; L/pTAg: purified antigen in liposomes; STAg: soluble tachyzoites alone; FCA/TAg: soluble tachyzoites with Freund's complete adjuvant FCA/PBS: Freund's complete adjuvant with PBS; L/PBS: liposomes with PBS; PBS: saline alone (control).

cysts were found only in mice immunized with antigen containing liposomes. There are statistically significant differences between the mice immunized with L/TAg, L/pTAg, L/TCAg and L/CAg and control mice inoculated with PBS, FCA and liposomes alone. Significant differences were also observed between L/TAg and TAg, as well as between L/TAg and FCA/TAg groups. On the other hand, there are no significant differences between the mice immunized with STAg and FCF/TAg when compared with the control groups.

\section{DISCUSSION}

We have described here a defined vaccine that results in protection against death in experimentally infected mice. Of importance here is the use of a purified antigen of tachyzoites with a cocktail of lifecycle stage-specific antigen (tachyzoites and tissue

TABLE II

Mean absorbance (Optical Density - OD) of mice sera before immunization and after the third immunization by Elisa and number of brain cysts

\begin{tabular}{lccc}
\hline Immunized mice & $\begin{array}{c}\text { OD } \pm \text { SD } \\
\text { before immunization }\end{array}$ & $\begin{array}{c}\text { OD } \pm \text { SD } \\
\text { after immunization }\end{array}$ & $\begin{array}{c}\text { Mean number of brain } \\
\text { cysts } \pm \text { SD }\end{array}$ \\
\hline L/TAg & $0.015 \pm 0.010$ & $0.219 \pm 0.033$ & $3322 \pm 690$ \\
L/CAg & $0.022 \pm 0.009$ & $0.131 \pm 0.067$ & $3031 \pm 1225$ \\
L/TCAg & $0.020 \pm 0.011$ & $0.351 \pm 0.096$ & $2330 \pm 1200$ \\
L/pTAg & $0.016 \pm 0.010$ & $0.180 \pm 0.016$ & $2410 \pm 752$ \\
STAg & $0.024 \pm 0.009$ & $0.199 \pm 0.086$ & $10916 \pm 3373$ \\
FCA/TAg & $0.023 \pm 0.009$ & $0.192 \pm 0.027$ & $7283 \pm 3772$ \\
FCA/PBS & $0.018 \pm 0.010$ & $0.025 \pm 0.008$ & $11666 \pm 3629$ \\
L/PBS & $0.017 \pm 0.009$ & $0.034 \pm 0.014$ & $13900 \pm 1819$ \\
PBS & $0.024 \pm 0.09$ & $0.014 \pm 0.004$ & $16466 \pm 8500$ \\
\hline
\end{tabular}

L/TAg: soluble tachyzoites antigen in liposomes; L/CAg: soluble tissue cysts in liposomes; L/TCAg: tachyzoites plus tissue cysts in liposomes; L/pTAg: purified antigen in liposomes; STAg: soluble tachyzoites alone; FCA/TAg: soluble tachyzoites with Freund's complete adjuvant; FCA/PBS: Freund's complete adjuvant with PBS; L/PBS: liposomes with PBS; PBS: saline alone (control). 
cysts) in immunization. The use of purified protein vaccine has been strengthened by three reports which have demonstrated that immunization with SAG 1, either in the presence of Quil A (Khan et al. 1988), incorporated into liposomes (Bulow \& Boothroyd 1991) or recombinant SAG1 expressed in E. coli (Petersen et al. 1998), confer an efficient protection to mice challenged with moderately virulent strains of $T$. gondii. Clearly, the specific immune response of the host will determine the outcome of post-challenge infection. Here, animals immunized with liposome entrapped soluble antigens and entrapped tachyzoite purified antigen showed similar levels of protection (between 70-100\%). The animals also showed low numbers of brain cysts when compared to the controls.

The incorporation of tachyzoite soluble antigens in liposomes increased significantly the degree of protection in terms of survival, as well as brain cyst development. However, no significant increase of antibody production was observed. These data suggest that liposomes exerted their adjuvant effect by stimulating cell-mediated immunity, but not humoral immunity. FCA, on the other hand, contrary to liposomes, did not show any significant immuno-adjuvant properties.

Another aspect we addressed in this study was the effect on vaccine efficacy of combining antigens of different stages. To do this, we compared groups immunized with tachyzoite soluble antigen and with tissue cyst-soluble antigen (both in the encapsulated form in liposomes) with a group immunized with a mixture of the antigens encapsulated in liposomes. Although the combination of both antigens ( $\mathrm{L} / \mathrm{TCAg})$ stimulated antibody production, increased survival, and reduced the mean number of cysts, the results were not statistically significant when compared to the two other groups (L/TAg and L/CAg).

The results obtained here, using a cocktail or a defined surface antigen, support the findings of McLeod et al. (1988), Escajadillo and Frenkel (1991), and Waldeland and Frenkel (1983), using dead parasites, and Overnes et al. (1991), and Sharma et al. (1983), using subunit vaccines incorporating primarily non-surface antigen. In all cases the target for a vaccine could be either the domestic animals (pigs, sheeps, goats and cats) from which the infection is readily passed to humans or seronegative pregnant women and immunodeficient/immunodepressed individuals.

By at least one criterion (survival), a cocktail of antigen and a defined, purified antigen-based vaccine can provide similar effective protection. As a further refinement, we suggest the need to test the use of an antigen cocktail or defined purified protein at each stage of $T$. gondii-infection.

\section{ACKNOWLEDGEMENT}

To Prof. Anthony Rylands for reviewing the manuscript.

\section{REFERENCES}

Alexander J, Roberts CW, Brewer JM 1993. Progress towards the development of a vaccine against congenital toxoplasmosis: identification of protective antigens and the selection of the appropriate adjuvants, p. 217-229. In JE Smith NATO-ASI on Toxoplasmosis, vol. H. 78, Elsevier Publications, Cambridge.

Araújo FG 1994. Immunization against Toxoplasma gondii. Parasitol Today 10: 358-360.

Bradford MM 1976. A rapid and sensitive method for the quantitation of microgram quantities of protein utilizing the principle of protein dye binding. Anal Biochem 72: 248-254.

Bulow R, Boothroyd JC 1991. Protection of mice from fatal Toxoplasma gondii infection by immunization with P30 antigen in liposomes. J Immunol 147: 246251.

Bulow R, Overath P 1986. Purification and characterization of the membrane-form variant surface glygoprotein hydrolase of Trypanosoma brucei. J Biol Chem 261: 11-18.

Buxton D 1993. Toxoplasmosis: the first commercial vaccine. Parasitol Today 9: 335-337.

Dubey JP, Barker DG, Davis SW, Urban JR 1994. Persistence of immunity to toxoplasmosis in pigs vaccinated with a nonpersistent strain of Toxoplasma gondii. Am J Res 55: 982-987.

Duncan DB 1955. Multiple range and multiple F tests. Biometrics 11: 1-42.

Escajadillo A, Frenkel JK 1991. Vaccine tests in Aotus monkeys. Am J Trop Med Hyg 44: 382-389.

Gazzinelli RT, Talvani A, Camargo MM, Santiago HC, Oliveira MAP, Vieira LQ, Martins GA, Aliberti JCS, Silva JS 1998. Induction of cell-mediated immunity during early stages of infection with intracellular protozoa, Brazil. J Med Biol Res 31: 89-104.

Jamra LMF, Vieira MPL 1991. Isolamento do Toxoplasma gondii de exsudato peritoneal e órgãos de camundongos com infecção experimental. Rev Inst Med Trop São Paulo 33: 435-441.

Johnson AM, Mcdonald PJ, Neoh SH 1983. Monoclonal antibodies to Toxoplasma cell membrane surface antigens protect mice from toxoplasmosis. $J$ Protozool 30: 351-356.

Kasper LH 1989. Identification of stage specific antigens of Toxoplasma gondii. Infec Immun 57: 668672.

Kasper LH, Currie KM, Bradley MS 1985. An unexpected response to vaccination with a purified major membrane tachyzoites antigen (P30) of Toxoplasma gondii. J Immunol 134: 3436-3451.

Khan IA, Smith JS, Kasper LH 1988. Induction of antigen-specific parasiticidal cytotoxic $\mathrm{T}$ cell splenocytes by a major membrane protein (P30) of Toxoplasma gondii. J Immunol 141: 3600-3606.

Kirby C, Gregoriadis G 1984. Dehydration-rehydration vesicles: a simple method for high yield drug entrapment in liposomes. Biotechnology 2: 979. 
Luft BJ, Hafner MD, Korzun AH, Leport C, Antoniskis D, Boster EM, Bourland III DD, Ultamchndani R, Fuhrer J, Jacobson J, Morlat P, Vilde J, Remington JS, Members of the ACTG 077p/ANRS 009 study team 1993. Toxoplasmic encephalitis in patients with the acquired immunodeficiency syndrome. $N$ Engl J Med 329: 995-1000.

McLeod R, Frenkel JR, Estes RG, Mack DG, Eisenhauer PB, Gibbons G 1988. Subcutaneous and intestinal vaccination with tachyzoites of Toxoplasma gondii and acquisition of immunity to peroral and congenital toxoplasma challenge. J Immunol 140: 16321637.

Nóbrega P, Trapp E, Giovannoni M 1952. Toxoplasmose epizótica em coelhos. I. Ação da sulfadiazina. Ciên Cult 4: 134-135.

Overnes G, Nesse LL, Waldeland H, Lövgren K, Gudding R 1991. Immune response after immunization with an experimental Toxoplasma gondii iscom vaccine. Vaccine 9: 25-28.
Petersen E, Nielsen HV, Christiansen L, Spenter J 1998. Immunization with $E$. coli produced recombinant $T$. gondii SAG1 with alum as adjuvant protect mice against lethal infection with Toxoplasma gondii. Vaccine 16: 1283-1289.

Sharma SD, Araújo FG, Remington JS 1983. Toxoplasma antigen isolated by affinity chromatography with monoclonal antibody protects mice against lethal infection with Toxoplasma gondii. Infect Immun 33: 2818-2820.

Waldeland H, Frenkel JK 1983. Live and killed vaccines against toxoplasmosis in mice. J Parasitol 69: 60-64.

Wong SY, Remington JS 1994. Toxoplasmosis in pregnancy. State of Art Clinical, Article. Clin Infect Dis 18: 853-862.

Yap GS, Scharton-kersten T, Ferguson DJP, Howe D, Suzuki Y, Sher A 1998. Partially protective vaccination permits the development of latency in a normally virulent strain of Toxoplasma gondii. Infect Immun 66: 4382-4388. 